\title{
Sidelobe Suppression Beamforming Using Tapered Amplitude Distribution for a Microwave Power Transfer System with a Planar Array Antenna
}

\author{
Inho Park $^{1} \cdot$ Chulhun Seo ${ }^{2} \cdot$ Hyunchul $\mathrm{Ku}^{1, *}$
}

\begin{abstract}
This paper proposes a sidelobe suppression beamforming scheme using tapered amplitude distribution for microwave power transfer (MPT) with a two-dimensional planar array antenna (PAA). To overcome the undesirable effects of sidelobes, the magnitude of the sidelobes can be lowered by manipulating the amplitude distribution of the PAA. By analyzing the array factor of the PAA, we expressed the amplitude distribution as the product of two one-dimensional vectors. Moreover, we developed a method for obtaining these vectors from an expected sidelobe level (SLL). We used the Chebyshev taper, which has a higher directivity than other distributions given an expected SLL, for the amplitude distribution. To verify the proposed method, we designed and implemented a digital beamforming system with a $4 \times 4$ transmitter $(\mathrm{Tx})$ array and a receiver $(\mathrm{Rx})$ at $5.8 \mathrm{GHz}$. Compared with a uniform amplitude distribution, the proposed method reduced the SLL by $7.5 \mathrm{~dB}$ but the received RF power by only $0.6 \mathrm{~dB}$. We compared the performance of the proposed MPT system with the results of previous studies.
\end{abstract}

Key Words: Array Factor, Digital Beamforming, Microwave Power Transfer, Sidelobe Suppression, Wireless Power Transfer.

\section{INTRODUCTION}

Microwave power transfer (MPT) is attracting attention for use in Internet of Things (IoT) and medical devices $[1,2]$ because it has the potential to transmit power over long distances, irrespective of the receiver's $(\mathrm{Rx})$ location. However, it also has the disadvantage of low efficiency due to path loss. To overcome this drawback, the transmitter $(\mathrm{Tx})$ configures a massive array antenna and increases the magnitude of the radiated signal per channel. Unfortunately, this approach intensifies the sidelobe, which can adversely affect the surrounding electronics, as well as human beings; therefore, it is necessary to lower the sidelobe level (SLL) while maintaining the magnitude of the main lobe.

Several studies have been conducted on high-efficiency MPT, such as retro-reflective beamforming [3, 4]. This technique, which has been investigated for radar applications, transfers power efficiently by tracking the location of the $\mathrm{Rx}$ through a pilot signal and transmitting power in the same direction. Other studies on MPT have used look-up tables (LUTs) [5]. In this method, phase values for an array antenna that can transmit power optimally in the direction of the designated $\mathrm{Rx}$ antenna are stored in an LUT, with the beamforming directed toward the located Rx antenna. Although [3-5] achieved high efficiency under ideal conditions, they did not consider lowering the SLL;

Manuscript received April 29, 2021 ; Revised June 4, 2021 ; Accepted June 15, 2021. (ID No. 20210429-046J)

${ }^{1}$ Department of Electronics Information and Communication Engineering, Konkuk University, Seoul, Korea.

${ }^{2}$ School of Electronic Engineering, Soongsil University, Seoul, Korea.

"Corresponding Author: Hyunchul Ku (e-mail: hcku@konkuk.ac.kr)

This is an Open-Access article distributed under the terms of the Creative Commons Attribution Non-Commercial License (http://creativecommons.org/licenses/by-nc/4.0) which permits unrestricted non-commercial use, distribution, and reproduction in any medium, provided the original work is properly cited.

(c) Copyright The Korean Institute of Electromagnetic Engineering and Science. 
hence, MPT studies aiming to reduce SLL are underway [6-9].

A few researchers have applied various techniques for the non-uniform distribution of the magnitude of signals from array antennas; for example, [6] studied this for the application of MPT to drones. In their simulation, the researchers suppressed the SLL to $-17.7 \mathrm{~dB}$ by applying a Gaussian function to the amplitude distribution of a $1 \times 14 \mathrm{Tx}$ array antenna at $5.8 \mathrm{GHz}$. In [7], the results for uniform amplitude distribution were compared to those for applying the Kaiser function to the amplitude distribution of an array antenna. The researchers verified through simulation that their technique reduced the SLL to $-12.8 \mathrm{~dB}$ at 9.5 GHz. In [8], the Taylor function was applied to the feeding network of an array antenna. The researchers fabricated a $1 \times 8$ array antenna at $28 \mathrm{GHz}$ and confirmed the reduction of SLL to $-15.2 \mathrm{~dB}$. Study [9] matched the weighting coefficient of the Chebyshev function to the feeding line impedance of an antenna. The researchers designed a $1 \times 5$ array antenna at $9.3 \mathrm{GHz}$ and verified the suppression of the SLL to $-17.4 \mathrm{~dB}$. However, the elements fabricated in these studies could only form one beam pattern for a fixed SLL. Different elements may be needed to achieve a minimum SLL for different beam directions. To overcome this drawback, we propose an adaptive beamforming system with sidelobe suppression that can actively form the desired beam.

This paper presents a method for lowering SLL by adjusting the amplitude distribution of a two-dimensional planar array antenna (PAA). The phase distribution of a signal applied to an array antenna determines the direction of the main beam, and the amplitude distribution determines the SLL, main beamwidth, and nulls [10]; therefore, to reduce SLL, the amplitude of the array antenna must be distributed non-uniformly. This study proposes a method for obtaining an amplitude distribution matrix with the weight of each antenna as an element in an $M \times$ $N$ PAA. This $M \times N$ matrix is derived as the product of the amplitude distribution vectors of two linear array antennas (LAAs) constituting the PAA. To lower the SLL while maintaining the total transmission power, we increased the amplitude of the signal applied to the central element of the array antenna and lowered the amplitude of the signal applied to the side element using the tapered amplitude distribution method [10]. Although this method reduces the sidelobe of the beam pattern, it weakens directivity owing to the widening of the beamwidth; therefore, we used the Chebyshev tapered amplitude distribution method, which is efficient and possesses the strongest directivity among distribution methods with the same maximum SLL [11]. Moreover, by limiting all sidelobes to the same level, we minimized the RF radiation power in all directions, except for the main lobe.

To verify the validity of the proposed method, we designed a digital beamforming system with a $4 \times 4 \mathrm{Tx}$ array and an $\mathrm{Rx}$ operating at $5.8 \mathrm{GHz}$. The Tx of the digital beamforming system was an adaptive device capable of generating signals with the desired phase and amplitude. Furthermore, for MPT, the Tx consisted of a digital Tx stage for RF signal generation and a power amplification (PA) stage. The magnitude of the signal from each channel of the Tx had a least significant bit (LSB) of $0.2 \mathrm{~dB}$, and the phase had an LSB of $0.36^{\circ}$. The maximum output power per channel was $1 \mathrm{~W}$. We conducted the measurements in the far field and verified the performance using the received power.

The remainder of this paper is organized as follows. Section II describes the sidelobe suppression method for the PAA. Section III explains the design, implementation, and simulation of the digital beamforming system for verification. Section IV conducts the experiments and discusses system performance. Finally, Section V presents the study's conclusions.

\section{SIDELOBE SUPPRESSION BEAMFORMING}

Fig. 1 shows a schematic diagram illustrating the relationship between sidelobe and directivity during beamforming. It depicts steering of the main beam in the direction for received power and sidelobe suppression in the direction of the person to reduce power. As mentioned in the previous section, the sidelobe can be altered by manipulating the amplitude distribution of the array antenna, but a nonuniform amplitude distribution can widen the main lobe's beamwidth [10], weakening the directivity of the array antenna. Directivity is the yardstick for power transmission in the intended direction; therefore, the SLL must be reduced as much as possible, and an amplitude distribution applied with high directivity. To this end, studies frequently use a tapered amplitude distribution to increase the amplitude of the central element antenna and decrease that of the side element antenna. The Chebyshev taper has the narrowest main lobe beamwidth, given a limited SLL [11]; therefore, we applied the

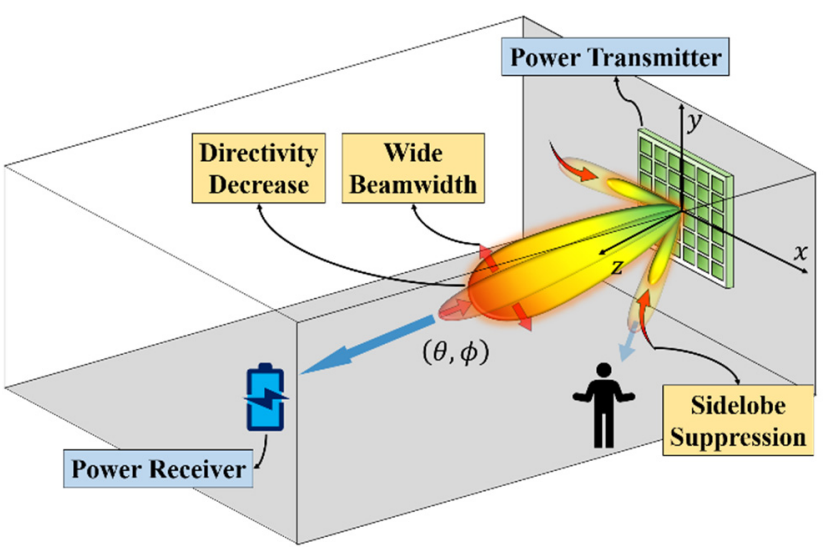

Fig. 1. Sidelobe suppression beamforming considering directivity and beamwidth. 
Chebyshev taper to the amplitude distribution through an array factor (AF) of far-field approximation.

Fig. 2 depicts the structure of the PAA. As shown in Fig. 2, the PAA consists of two intersecting LAAs, and AF is equal to the product of the AFs of the two LAAs. The AFs of the two LAAs are as follows [10]:

$$
\begin{gathered}
A F_{x}(\theta, \phi)=\sum_{m=1}^{M} a_{m} e^{j(m-1)\left(k d_{x} \sin \theta \cos \phi+\psi_{x}\right)} \\
A F_{y}(\theta, \phi)=\sum_{m=1}^{N} b_{n} e^{j(n-1)\left(k d_{y} \sin \theta \sin \phi+\psi_{y}\right)}
\end{gathered}
$$

where $a_{m}$ and $b_{n}$ are the amplitude distributions of the $x$ - and $y$ axis LAAs, $M$ and $N$ are the numbers of $x$-axis and $y$-axis array elements, $d_{x}$ and $d_{y}$ are the spacings between the elements of the $x$-axis and $y$-axis LAAs, and $\psi_{x}$ and $\psi_{y}$ are the phases of the $x$ axis and $y$-axis LAAs, respectively. $k$ is the wave number, $\theta$ is the elevation angle, and $\phi$ is the azimuth angle. The amplitude distributions of the two axes, $a_{m}$ and $b_{n}$, can be expressed as $1 \times M$ and $1 \times N$ vectors, respectively,

$$
\begin{aligned}
\boldsymbol{a} & =\left[\begin{array}{llllll}
a_{1} & a_{2} & \cdots & a_{m} & \cdots & a_{M}
\end{array}\right] \\
\boldsymbol{b} & =\left[\begin{array}{llllll}
b_{1} & b_{2} & \cdots & b_{n} & \cdots & b_{N}
\end{array}\right]
\end{aligned}
$$

Furthermore, the AF of the PAA can be expressed by the product of Eqs. (1) and (2), as follows:

$$
\begin{aligned}
& A F(\theta, \phi)=A F_{x}(\theta, \phi) A F_{y}(\theta, \phi) \\
&=\sum_{n=1}^{N}\left[\sum_{m=1}^{M} w_{m, n} e^{j(m-1)\left(k d_{x} \sin \theta \cos \phi+\psi_{x}\right)}\right] \\
& e^{j(n-1)\left(k d_{y} \sin \theta \sin \phi+\psi_{y}\right)}
\end{aligned}
$$

where $w_{m, n}$ is the product of $a_{m}$ and $b_{n}$. An $M \times N$ matrix with $w_{m, n}$, which is the amplitude distribution of the PAA, can then be expressed as follows:

$$
\boldsymbol{W}=\boldsymbol{a}^{T} \boldsymbol{b}
$$

To obtain Eq. (6), we analyzed the AFs of the LAA for the two axes. Since the phase and amplitude distributions are independent of each other in this process of obtaining the amplitude dis-

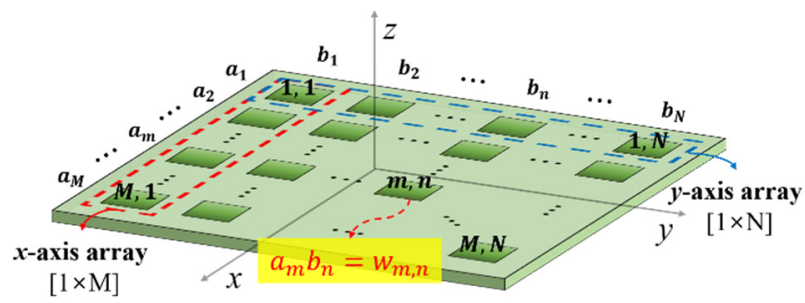

Fig. 2. Geometry structure of the planar array antenna. tribution, the phase $\left(\psi_{x}\right.$ and $\left.\psi_{y}\right)$ that determines the beam direction can be considered a constant; therefore, in Eq. (1), the AF of the $x$-axis LAA is a series of exponential functions that can be expressed as follows:

$$
A F_{x}(\theta, \phi)=\sum_{m=1}^{M} a_{m} z^{(m-1)}
$$

where $z=e^{j\left(k d_{x} \sin \theta \cos \phi+\psi_{x}\right)}$. Eq. (7) is the same as the $(M-1)^{\text {th }}$ order equation, and can therefore be rewritten as

$$
\begin{aligned}
A F_{x}(\theta, \phi) & =a_{1}+a_{2} z+\cdots+a_{M} z^{M-1} \\
& =a_{M}\left(\frac{a_{1}}{a_{M}}+\frac{a_{2}}{a_{M}} z+\cdots+z^{M-1}\right)
\end{aligned}
$$

Because Eq. (8) is a polynomial of the degree $(M-1)$ equation, it can be expressed as a product of $(M-1)$ linear terms, as follows:

$$
\begin{gathered}
A F_{x}(\theta, \phi)=a_{M}\left\{\prod_{i=1}^{M-1}\left(z-z_{i}\right)\right\} \\
z_{i}=e^{j \beta_{i}}
\end{gathered}
$$

where $z_{i}$ are the roots in the $\mathrm{AF}, \beta_{i}$ are the null directions, and $i$ ranges from 1 to $M-1$; hence, if the null directions are known, the roots of the $\mathrm{AF}$ can be found, and the $\mathrm{AF}$ expressed as the product of linear terms can be completed. The AF equation, which is the product of a linear term, is developed in polynomial form, and the coefficients of each term are mapped to the amplitude coefficients of the array antenna; hence, it describes the process for finding the null directions by mapping the Chebyshev polynomial [11] using the array factor. To this end, the difference in magnitude between the main lobe and the sidelobe is defined as follows:

$$
S=20 \log _{10}\left|\frac{A F_{x}\left(\theta_{S L}, \phi_{S L}\right)}{A F_{x}\left(\theta_{M L}, \phi_{M L}\right)}\right|
$$

where $\left(\theta_{M L}, \phi_{M L}\right)$ is the main lobe direction and $\left(\theta_{S L}, \phi_{S L}\right)$ is the sidelobe direction. We then derived the null directions $\left(\beta_{i}\right)$ using the following equations:

$$
\beta_{i}=2 \cos ^{-1}\left\{\frac{\cos \left(\frac{(2 i-1) \pi}{2 M-2}\right)}{\cosh \left(\frac{\pi A}{M-1}\right)}\right\}
$$

and

$$
A=\frac{1}{\pi} \cosh ^{-1}\left(10^{|S|}\right)
$$

This process is explained in detail in [12]. 
To determine the roots of the $\mathrm{AF}$, null directions obtained through Eq. (12) were assigned to Eq. (10), and Eq. (9) was expanded as follows:

$$
\begin{aligned}
A F_{x}(\theta, \phi) & =a_{M}\left(z-e^{j \beta}\right)\left(z-e^{j \beta_{2}}\right) \cdots\left(z-e^{j \beta_{M-1}}\right) \\
& =a_{M}\left(w_{x 1}+w_{x 2} z+\cdots+z^{M-1}\right)
\end{aligned}
$$

where $w_{x p}$ is the weight of the $p^{\text {th }}$ order term, and $p$ ranges from 1 to $M-1$. If $a_{M}$ is a coefficient that determines the overall magnitude of the amplitude distribution, then the coefficient of each order $\left(w_{x p}\right)$ can be expressed as a ratio; therefore, the amplitude distribution vector $(1 \times M)$ can be expressed as follows:

$$
\boldsymbol{a}=a_{M}\left[\begin{array}{lllll}
w_{x 1} & w_{x 2} & \cdots & w_{x(M-1)} & 1
\end{array}\right]
$$

Applying Eqs. (7)-(14) to the $y$-axis LAA yields the following vector:

$$
\boldsymbol{b}=b_{N}\left[\begin{array}{lllll}
w_{y 1} & w_{y 2} & \cdots & w_{y(N-1)} & 1
\end{array}\right]
$$

where $w_{y q}$ is the weight of $q^{\text {th }}$ order term, and $q$ ranges from 1 to $N-1$.

The coefficients of the linear vector in Eqs. (15) and (16) are the same as those of the Chebyshev taper, gradually increasing from the side element of the vector to the central element; hence, if Eqs. (15) and (16) are applied as weights of the array antenna, the radiated power from the side element antenna is low, and the radiated power from the central element antenna is high. This technique is often used to determine the weights in array antennas to form low sidelobes [9]. In this study, we need to know the amplitude distribution to be applied to the PAA; therefore, $W$, which is the Chebyshev tapered amplitude distribution for the $M \times N$ PAA, could be obtained through Eq. (6).
This process ends by mapping the elements of $W$ to the amplitude weights of each element of the PAA, allowing the realization of beamforming with an expected SLL. Fig. 3 illustrates the process flowchart for obtaining the Chebyshev tapered amplitude distribution for sidelobe suppression beamforming.

\section{SYSTEM DESIGN AND IMPLEMENTATION}

\section{System Design}

To verify the proposed sidelobe suppression scheme, we designed a digital beamforming system with a $\mathrm{Tx}$ array and an $\mathrm{Rx}$ operating at $5.8 \mathrm{GHz}$. Fig. 4 shows a block diagram of the system.

The Tx is a $4 \times 4$ array with 16 channels, a digital Tx stage, and a PA stage. The digital Tx stage generates an in-phase and

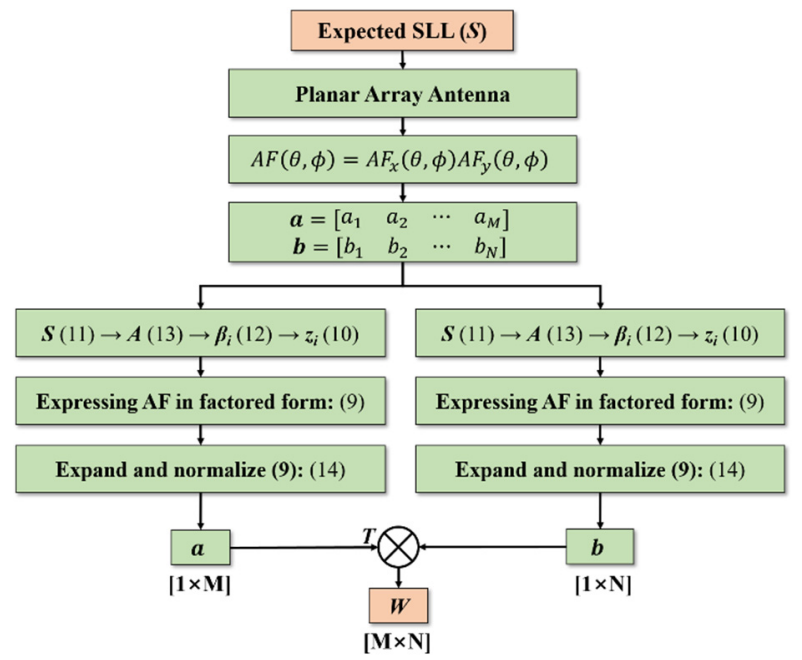

Fig. 3. Flowchart of the process for finding the amplitude distribution of the PAA for sidelobe suppression beamforming.

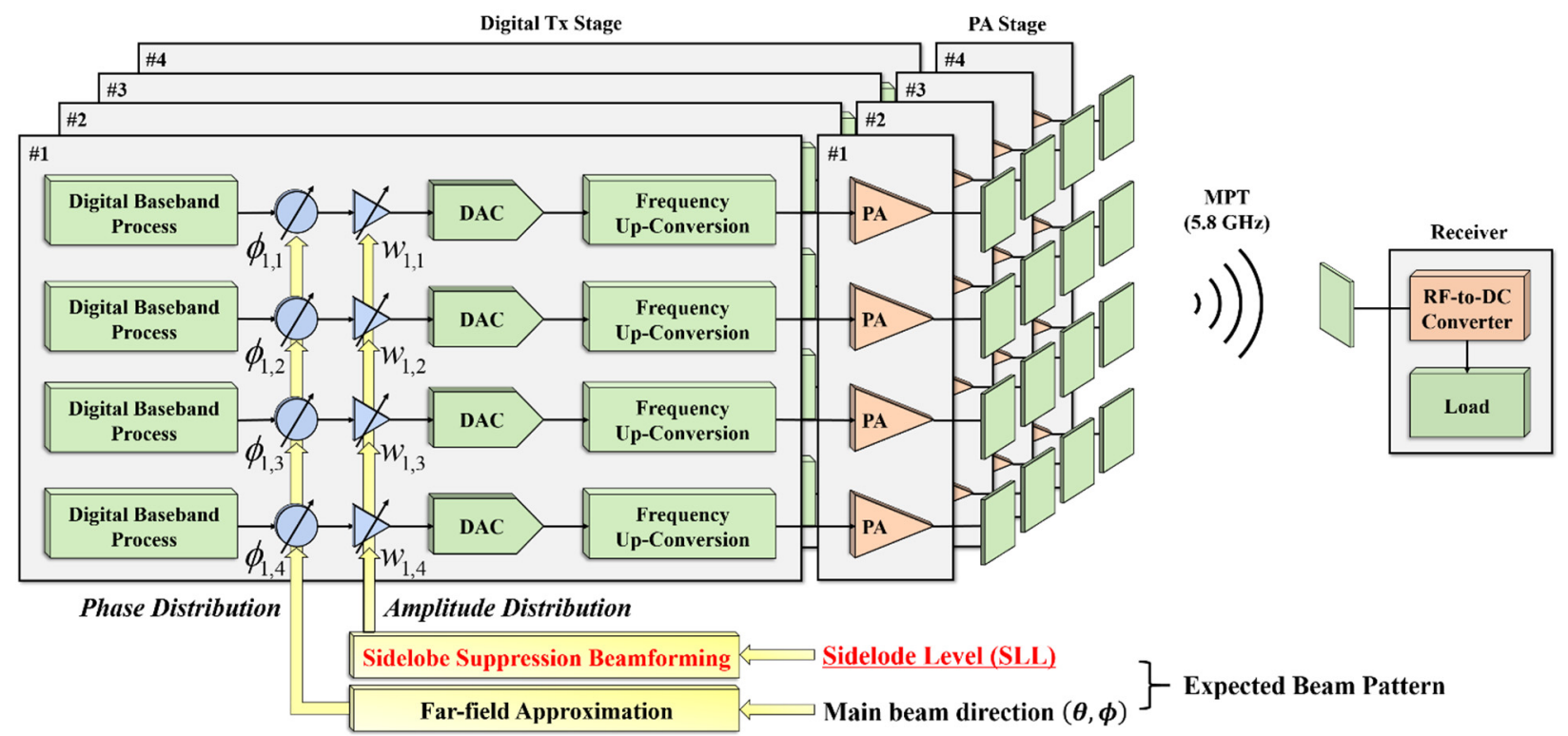

Fig. 4. Block diagram of the digital beamforming system for MPT. 
quadrature $(\mathrm{I} / \mathrm{Q})$ signal, which is a baseband signal, and converts it to an analog signal through a digital-to-analog converter. We adjusted the I/Q signal using the sidelobe suppression beamforming method described in the previous section based on an LSB gain of $0.2 \mathrm{~dB}$ and an LSB phase of $0.36^{\circ}$. The analog baseband signal is converted into an RF signal through frequency upconversion and output. The RF signal is then applied to the PA stage of the MPT, and the signal is amplified. The PA stage uses a PA with a power gain of $32 \mathrm{~dB}$ and a $\mathrm{P} 1 \mathrm{~dB}$ of 33 $\mathrm{dBm}$. The amplified $\mathrm{RF}$ signal radiates from a channel via a single antenna with a directivity of $9.3 \mathrm{dBi}$.

The $\mathrm{Rx}$ is composed of a single antenna, an RF-to-DC converter, and a load. The RF signal applied to the antenna is rectified to a DC signal by the RF-to-DC converter circuit. The $\mathrm{Rx}$ antenna is the same as a single Tx antenna with a directivity of $9.3 \mathrm{dBi}$.

Fig. 5 illustrates the antenna structure used in the digital beamforming system. The single antenna comprises a $2 \times 2$ sub-array with a single input port to increase the gain, as shown in Fig. 5. The feeding lines reaching each sub-antenna are designed identically, so that an in-phase signal is applied to or radiated from the sub-antenna. The substrate is made of a 0.8$\mathrm{mm}$-thick RF-35 laminate with a dielectric constant of 3.5 and a loss tangent of 0.002 . It has a reflection coefficient of approximately $-30 \mathrm{~dB}$ at $5.8 \mathrm{GHz}$, and it forms a vertically polarized $\mathrm{RF}$ signal.

Furthermore, the single antenna has a directivity of $9.3 \mathrm{dBi}$, and a $4 \times 4$ uniform array has 20.9 dBi. Fig. 6 shows the beam patterns simulated using AWR Microwave Office for the $z x$ and $z y$ planes, respectively.

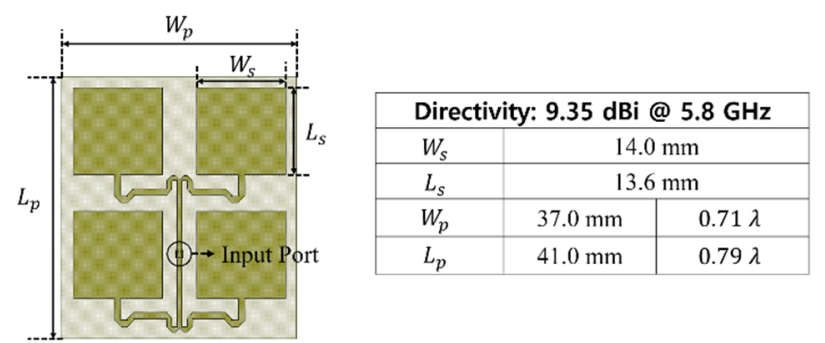

Fig. 5. Structure of the single antenna with $2 \times 2$ sub-array.

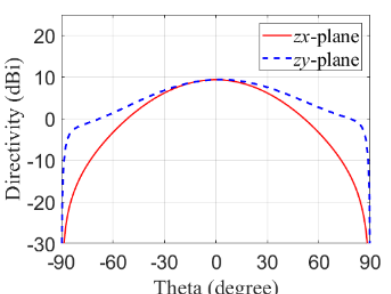

(a)

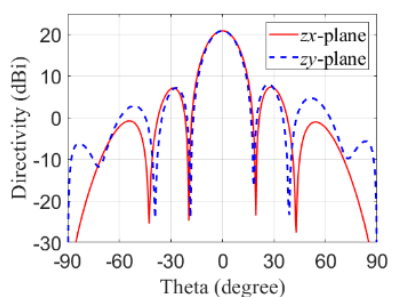

(b)
Fig. 6. Simulated beam patterns: (a) single antenna and (b) array antenna.

\section{Simulation}

To verify the proposed sidelobe suppression method based on the designed digital beamforming system, we simulated the method. To incorporate the trade-off between the sidelobe and the directivity mentioned in Section II into the simulation, we had to analyze the mathematical relationship between the two. The tapered amplitude distribution of the LAA can be expressed as the efficiency in terms of directivity, as follows [12]:

$$
\begin{aligned}
\eta_{T} & =\frac{D_{\text {taper }}}{D_{\text {uniform }}} \\
= & \frac{\left|\sum_{m=1}^{L} w_{m}\right|^{2} \sum_{n=1}^{L} \sum_{m=1}^{L} \operatorname{sinc}[(n-m) k d]}{L^{2} \sum_{n=1}^{L} \sum_{m=1}^{L} w_{n} w_{m}^{*} \operatorname{sinc}[(n-m) k d]}
\end{aligned}
$$

where $D_{\text {taper }}$ is the directivity of the tapered array, $D_{\text {uniform }}$ is the directivity of the uniform array, $w_{m}$ is the amplitude distribution, $d$ is the distance between elements, and $L$ is the number of antenna elements. The amplitude distribution for the beam pattern with the expected SLL can be obtained using the scheme proposed in Section II. By applying the obtained amplitude distribution, the formed beam can be expressed as the efficiency of directivity. Fig. 7 shows the taper efficiency and normalized amplitude weights for array antennas with different antenna spacings on the $x$ and $y$ axes, as explained in the previous subsection (Section III-1).

As shown in Fig. 7, the taper efficiency $\left(\eta_{T}\right)$ and the normalized amplitude of the central and side elements of the array antenna were presented according to $S$, which is the SLL. As $S$ increases, the taper efficiency is saturated at a specific value, and the amplitude deviation of the central and side elements is also saturated. Therefore, we studied three cases: one for the uniform amplitude distribution $\left(W_{1}\right)$, another for a taper efficiency of 0.9 $\left(W_{2}\right)$, and the last for the moment of entering the region within $5 \%$ of the saturation section of the efficiency curve $\left(W_{3}\right)$. Table 1 lists the SLL, amplitude distribution matrix $(a, b)$ and taper efficiency $\left(\eta_{T}\right)$ for each case. Fig. 8 shows the directivity when the amplitude distributions for each case were applied to the designed array antenna.

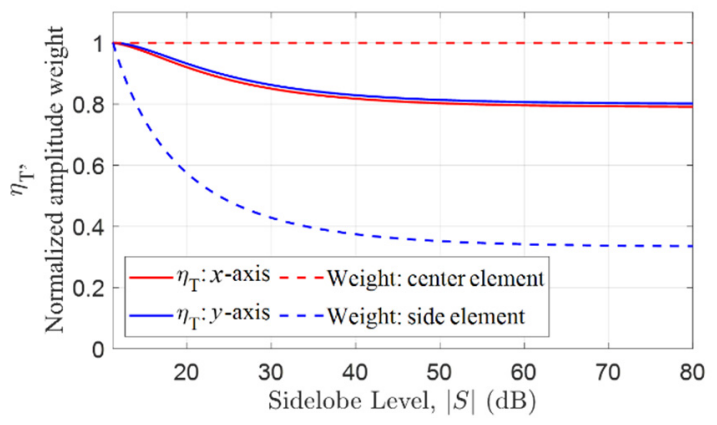

Fig. 7. Taper efficiency and normalized amplitude weight. 
Table 1. Parameters of three amplitude distributions

\begin{tabular}{lcccccccc}
\hline & \multicolumn{2}{c}{$W_{1}$} & & \multicolumn{2}{c}{$W_{2}$} & & \multicolumn{2}{c}{$W_{3}$} \\
\cline { 2 - 5 } \cline { 8 - 9 } \cline { 8 - 9 } & $x$-axis & $y$-axis & & $x$-axis & $y$-axis & & $x$-axis & $y$-axis \\
\hline$S(\mathrm{~dB})$ & N/A & N/A & & -23 & -24 & & -50 & -54 \\
$a, b$ & 1.000 & 1.000 & & 0.514 & 0.498 & & 0.352 & 0.347 \\
& 1.000 & 1.000 & & 1.000 & 1.000 & & 1.000 & 1.000 \\
& 1.000 & 1.000 & & 1.000 & 1.000 & & 1.000 & 1.000 \\
& 1.000 & 1.000 & & 0.514 & 0.498 & & 0.352 & 0.347 \\
$\eta_{T}$ & \multicolumn{3}{c}{1.0} & & \multicolumn{2}{c}{0.9} & & \multicolumn{2}{c}{0.81} \\
\hline
\end{tabular}

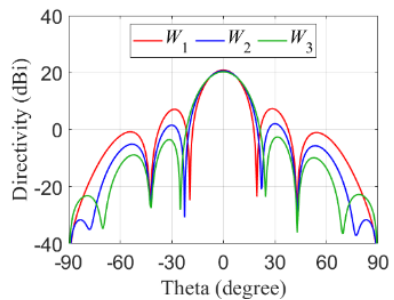

(a)

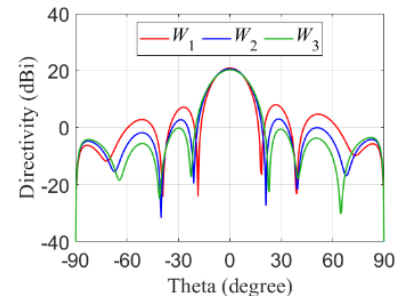

(b)
Fig. 8. Simulated array antenna directivity of three amplitude distributions: (a) $z x$-plane and (b) $z y$-plane.

\section{Implementation}

Fig. 9 shows a photograph of the implemented Tx. Fig. 9(a) depicts a digital $\mathrm{Tx}$ stage composed of RF transceivers, microcomputers to drive them, and a clock distribution device. It also has a port for supplying power and a LAN port at the back, controlled via LabVIEW on an external PC. Fig. 9(b) depicts a PA stage with a $1 \times 4$ array PA consisting of four layers. The antenna was mounted directly on the output terminal. The digital $\mathrm{Tx}_{\mathrm{x}}$ stage and PA stage were connected via coaxial cables.
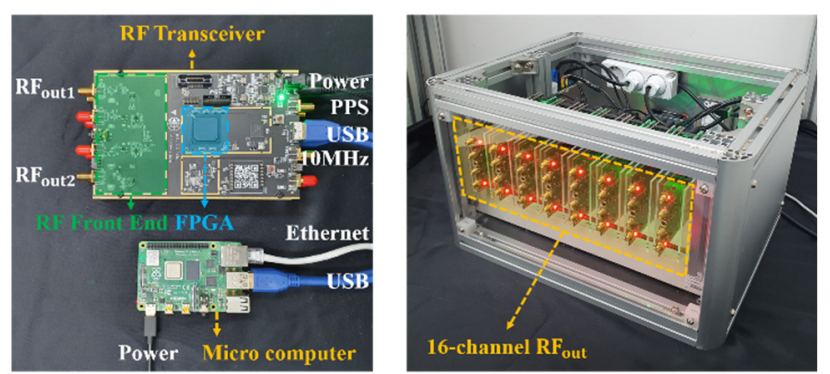

(a)
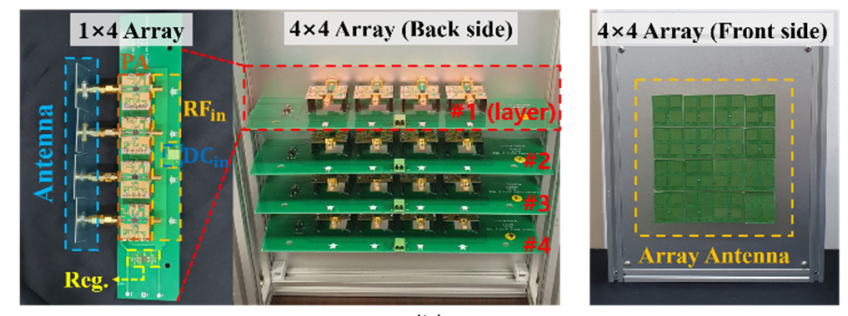

(b)

Fig. 9. Implemented $4 \times 4 \mathrm{Tx}$ array: (a) digital $\mathrm{Tx}$ stage and (b) PA stage.
We used USRP B210 (a software-defined radio) as the RF transceiver and a CDA-2990 clock distribution device. We used Raspberry Pi 4B as the microcomputer, and QPA9501 as the PA.

Fig. 10 shows a photograph of the $\mathrm{Rx}$. A single antenna, directional coupler, and RF-to-DC converter circuit were connected via the RF adapter. The RF signal collected by the $R x$ antenna was applied to the input port of a directional coupler (Mini-Circuits ZGBDC35-93HP+) to simultaneously measure the $\mathrm{RF}$ and rectified $\mathrm{DC}$ power. The power was measured through the coupling port, and the output RF signal was rectified to a DC signal by the RF-to-DC converter connected directly to the output port. The RF-to-DC converter circuit had an embedded Schottky diode (BAT68-04W) with a voltage doubler. The power conversion efficiency of the circuit was $59.5 \%$, which resulted from a rectified DC voltage of $6.5 \mathrm{~V}$ at an RF input power of $20 \mathrm{dBm}$ and a load resistance of $600 \Omega$.

The reflection coefficient $\left(S_{11}\right)$ of the single antenna was $-29.8 \mathrm{~dB}$, measured with a network analyzer (Keysight P5007A), and the directivity of the $4 \times 4$ array antenna was determined as $21 \mathrm{dBi}$.

\section{EXPERIMENTAL VERIFICATION}

Fig. 11 shows a photograph of the experimental setup for sidelobe suppression beamforming. The $\mathrm{Tx}$ was connected to the PC to control the beamforming system. The Rx was connected to a spectrum analyzer (Anritsu MS2713E) to measure the RF power and an electronic load (Keithley 2380-120-60) to measure the DC power. The gain of the transmitted RF signal was adjusted based on the three amplitude distributions obtained in Section III-2. The total RF power from the array antenna

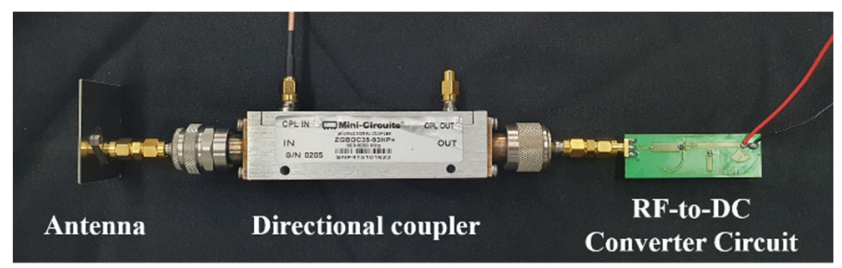

Fig. 10. Implemented Rx.

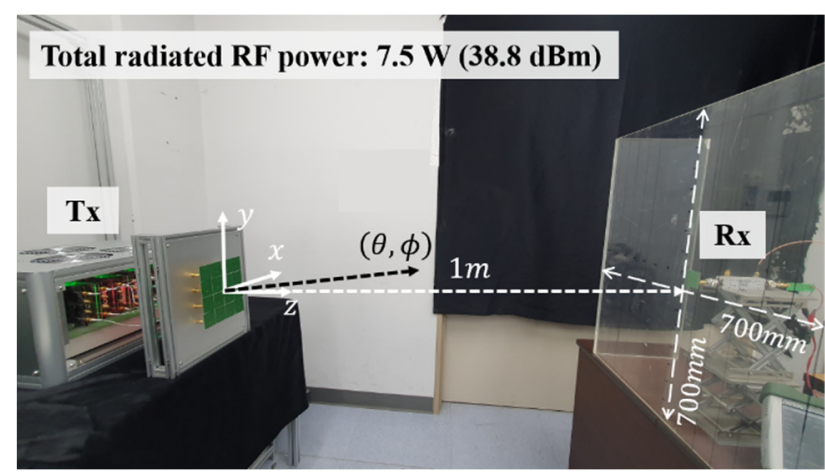

Fig. 11. Experimental setup for sidelobe suppression beamforming. 
was fixed at $7.5 \mathrm{~W}(38.8 \mathrm{dBm})$ in all cases.

Two experiments were conducted to verify the performance of the beamforming system. In the first, the radiation patterns of the $z x$ and $z y$ planes were measured. As shown in Fig. 11, this was based on the relative power by rotating the $T x$ array antenna in place while fixing the position of the Rx at the center. Fig. 12 illustrates the normalized radiation pattern plotted by measuring the received RF power from $-90^{\circ}$ to resemble that shown in Fig. 8, which resulted from the simulation. Compared with the SLL with $W_{1}$, which was a uniform distribution, the SLL while applying $W_{3}$ decreased by $7.5 \mathrm{~dB}$ from $-11.6 \mathrm{~dB}$ to $-19.1 \mathrm{~dB}$ on the $z x$ plane and $4.4 \mathrm{~dB}$ from $-11.5 \mathrm{~dB}$ to $-15.9 \mathrm{~dB}$ on the $z y$ plane. However, we confirmed that the received RF power at the central position $\left(0^{\circ}\right)$ decreased by only $0.6 \mathrm{~dB}$, from 21.0 $\mathrm{dBm}$ to $20.4 \mathrm{dBm}$. Furthermore, we verified that the rectified DC power also decreased by only $9.3 \mathrm{~mW}$, from $67.8 \mathrm{~mW}$ to $58.5 \mathrm{~mW}$.

In the second experiment, we verified the performance of beamforming in specific directions. As depicted in Fig. 11, the received $\mathrm{RF}$ power of $15 \times 15$ points was measured on the plane $\left(700 \times 700 \mathrm{~mm}^{2}\right)$ where the $\mathrm{Rx}$ could exist. The phase values for each channel of the main beam direction were adjusted by (5), giving the AF of the far field approximation. The main beam direction was set as $(\theta, \phi)$, with the center of the $\mathrm{Tx}$ array antenna as the origin in spherical coordinate systems. The experiment was conducted for three main beam directions, when $(\theta, \phi)$ was $\left(0^{\circ}, 0^{\circ}\right),\left(20^{\circ}, 135^{\circ}\right)$, and $\left(20^{\circ}, 220^{\circ}\right)$, respectively. Therefore, nine experiments in total were conducted for the three cases of amplitude distribution and three cases of phase distribution. Table 2 shows the distributions except for $W_{1}$ and $\left(0^{\circ}, 0^{\circ}\right)$ where all the amplitudes and phases of the channels were identical. Fig. 13 shows a color map obtained by measuring the $15 \times 15$ points of normalized received $\mathrm{RF}$ power for the nine experiments. We confirmed that the region with the sidelobe had smaller power in $W_{2}$ and $W_{3}$ than in $W_{1}$. Additionally, we verified the widening of the main lobe with an increase in the area occupied by power in the direction of the main lobe. Table 3 summarizes the results of the second experiment, including the received RF power and SLL for the nine measurements.

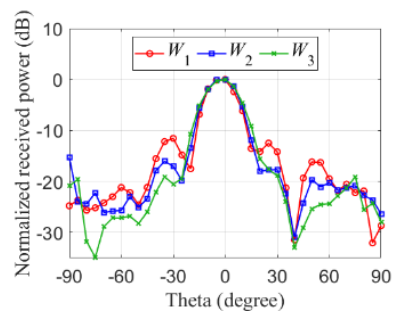

(a)

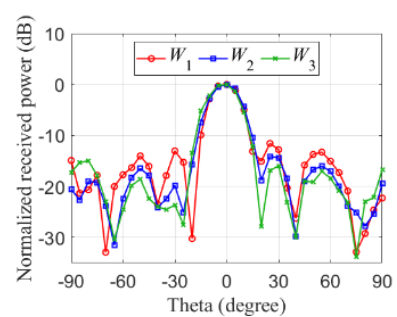

(b)
Fig. 12. Measured radiation patterns of three amplitude distributions: (a) $z x$-plane, (b) $z y$-plane.
Table 2. Amplitude and phase distributions for $4 \times 4$ array

\begin{tabular}{|c|c|c|c|c|c|}
\hline & \multirow{2}{*}{$\begin{array}{c}x \text {-axis } \\
\text { array index }\end{array}$} & \multicolumn{4}{|c|}{$y$-axis array index } \\
\hline & & 1 & 2 & 3 & 4 \\
\hline \multirow{8}{*}{$\begin{array}{l}\text { Amplitude } \\
\text { distribution, } \\
\mathrm{W}_{2} / \mathrm{W}_{3}\end{array}$} & 1 & $0.256 /$ & $0.514 /$ & $0.514 /$ & $0.256 /$ \\
\hline & & 0.122 & 0.352 & 0.352 & 0.122 \\
\hline & 2 & $0.498 /$ & $1.000 /$ & $1.000 /$ & $0.498 /$ \\
\hline & & 0.347 & 1.000 & 1.000 & 0.347 \\
\hline & 3 & $0.498 /$ & $1.000 /$ & $1.000 /$ & $0.498 /$ \\
\hline & & 0.347 & 1.000 & 1.000 & 0.347 \\
\hline & 4 & $0.256 /$ & $0.514 /$ & $0.514 /$ & $0.256 /$ \\
\hline & & 0.122 & 0.352 & 0.352 & 0.122 \\
\hline \multirow{8}{*}{$\begin{array}{l}\text { Phase distribution, } \\
\left(20^{\circ}, 135^{\circ}\right) / \\
\left(20^{\circ}, 220^{\circ}\right)\end{array}$} & 1 & $350.0^{\circ} /$ & $285.9^{\circ} /$ & $222.0^{\circ} /$ & $158.0^{\circ} /$ \\
\hline & & $200.3^{\circ}$ & $131.0^{\circ}$ & $61.8^{\circ}$ & $352.5^{\circ}$ \\
\hline & 2 & $60.6^{\circ} /$ & $356.6^{\circ} /$ & $292.7^{\circ} /$ & $228.7^{\circ} /$ \\
\hline & & $136.1^{\circ}$ & $66.8^{\circ}$ & $357.5^{\circ}$ & $288.2^{\circ}$ \\
\hline & 3 & $131.3^{\circ} /$ & $67.3^{\circ} /$ & $3.4^{\circ} /$ & $299.4^{\circ} /$ \\
\hline & & $71.8^{\circ}$ & $2.5^{\circ}$ & $293.2^{\circ}$ & $223.9^{\circ}$ \\
\hline & 4 & $202.0^{\circ} /$ & $138.0^{\circ} /$ & $74.1^{\circ} /$ & $10.1^{\circ} /$ \\
\hline & & $7.5^{\circ}$ & $298.2^{\circ}$ & $229.0^{\circ}$ & $159.7^{\circ}$ \\
\hline
\end{tabular}

Furthermore, we calculated $\Delta$ power and $\Delta \mathrm{SLL}$ (the differences between the received power and the SLL) from the uniform amplitude distribution (i.e., $\left.W_{1}\right)$. In $\left(0^{\circ}, 0^{\circ}\right)$-the case with central beamforming-received power was the same as in the first experiment, but the SLL decreased further. This occurred because the origin of the measured $15 \times 15$ points was $1 \mathrm{~m}$ away from the $\mathrm{Tx}$, but the remaining points were at greater distances than $1 \mathrm{~m}$, meaning that the received power was lower. Therefore, in the case of beamforming toward the center, we confirmed $\triangle \mathrm{SLL}$ to be $9.3 \mathrm{~dB}$ at the maximum. In $\left(20^{\circ}, 135^{\circ}\right)$ - the case with the second direction- $\triangle \mathrm{SLL}$ and $\Delta$ power were $5.9 \mathrm{~dB}$ and $0.8 \mathrm{~dB}$ in $W_{3}$, respectively. Additionally, in $\left(20^{\circ}, 220^{\circ}\right)$ - the case with the third direction- $\Delta \mathrm{SLL}$ and $\Delta$ power were $6.8 \mathrm{~dB}$ and $0.5 \mathrm{~dB}$ in $W_{3}$, respectively. These results proved that the received power barely decreased and that the SLL decreased significantly.

The experimental results compared with other studies on MPT are listed in Table 4. As mentioned earlier, the comparison was based on the beam pattern of the $z x$ plane in Fig. 12, which resulted from the first experiment. Studies [3-5] focused on the beamforming technique without considering lowering the sidelobe, and, in most cases, demonstrated high SLL. Additionally, [6-9] designed and manufactured a Tx device using a technique to lower the SLL, but different elements may be required to achieve a minimum SLL for other beam directions. The proposed sidelobe suppression beamforming system is suitable for safe wireless power transmission with high received power and low SLL. 

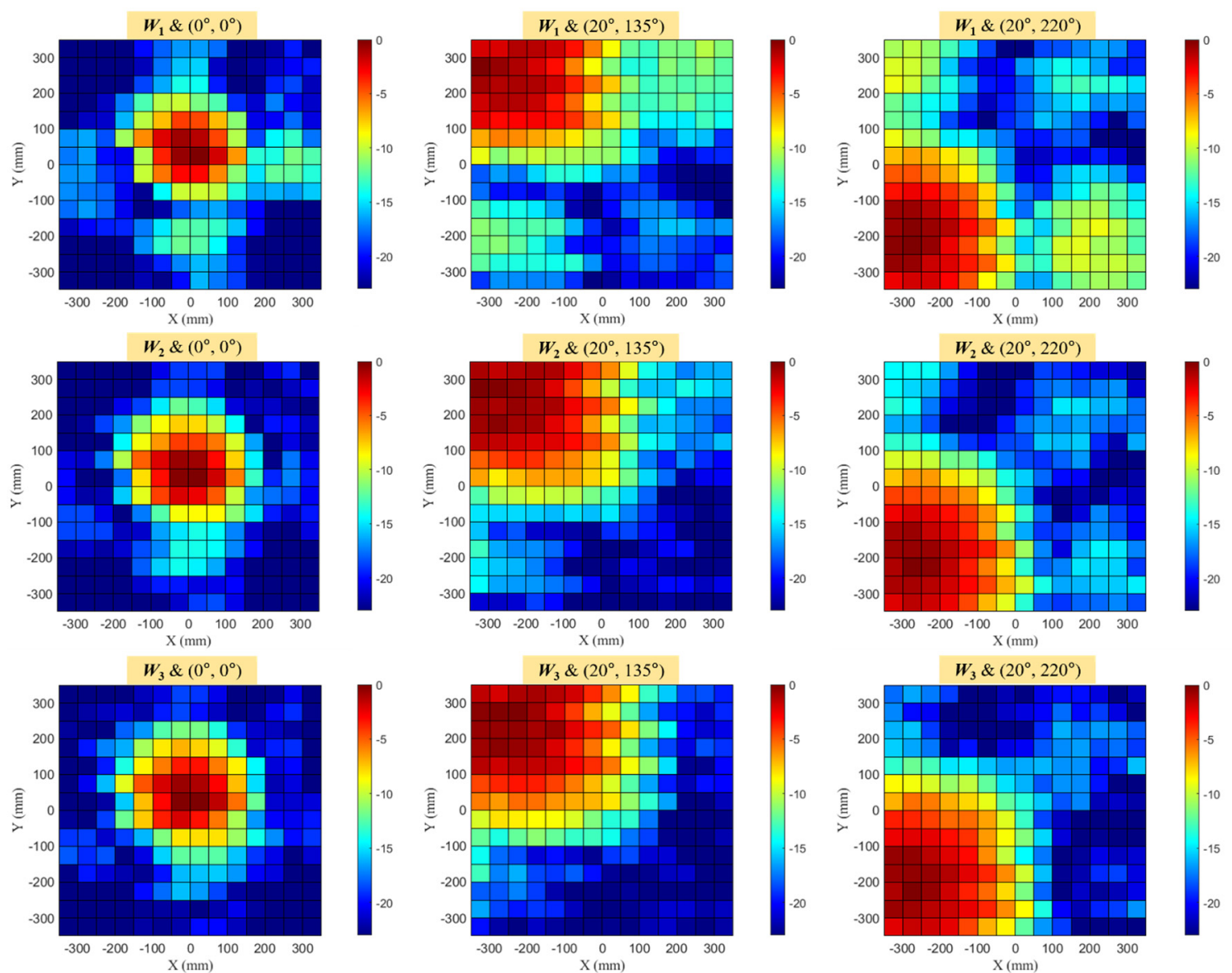

Fig. 13. Color maps of normalized received RF power in the beamforming experiments for three amplitude and phase distributions.

Table 3. Results of beamforming experiments for three amplitude and phase distributions

\begin{tabular}{|c|c|c|c|c|c|c|}
\hline \multirow{3}{*}{$\begin{array}{l}\text { Amplitude } \\
\text { distribution }\end{array}$} & \multicolumn{6}{|c|}{ Main beam direction $(\theta, \phi)$} \\
\hline & \multicolumn{2}{|c|}{$\left(0^{\circ}, 0^{\circ}\right)$} & \multicolumn{2}{|c|}{$\left(20^{\circ}, 135^{\circ}\right)$} & \multicolumn{2}{|c|}{$\left(20^{\circ}, 220^{\circ}\right)$} \\
\hline & $\begin{array}{c}\text { Received RF power } \\
\quad(\Delta \text { power })\end{array}$ & $\begin{array}{c}\text { SLL } \\
(\Delta \mathrm{SLL})\end{array}$ & $\begin{array}{c}\text { Received RF power } \\
\quad(\Delta \text { power })\end{array}$ & $\begin{array}{c}\text { SLL } \\
(\Delta \mathrm{SLL})\end{array}$ & $\begin{array}{c}\text { Received RF power } \\
\quad(\Delta \text { power })\end{array}$ & $\begin{array}{c}\text { SLL } \\
(\Delta \mathrm{SLL})\end{array}$ \\
\hline$W_{1}$ & $21.0(0)$ & $-11.7(0)$ & $18.9(0)$ & $-10.3(0)$ & $18.3(0)$ & $-9.0(0)$ \\
\hline$W_{2}$ & $20.7(0.3)$ & $-17.1(5.4)$ & $18.4(0.5)$ & $-13.0(2.7)$ & $18.1(0.2)$ & $-14.3(5.3)$ \\
\hline$W_{3}$ & $20.4(0.6)$ & $-21.0(9.3)$ & $18.1(0.8)$ & $-16.2(5.9)$ & $17.8(0.5)$ & $-15.8(6.8)$ \\
\hline
\end{tabular}

Total transmitted RF power: $7.5 \mathrm{~W}(38.8 \mathrm{dBm})$.

Received RF power is measured in $\mathrm{dBm}$; SLL and the differences between the received power and the SLL are measured in dB.

Table 4. Comparison with other studies

\begin{tabular}{lccccc}
\hline \multicolumn{1}{c}{ Study } & $\begin{array}{c}\text { Amplitude } \\
\text { distribution }\end{array}$ & Experimental setup / Beamforming system / Technique & $\begin{array}{c}\text { Freq. } \\
(\mathrm{GHz})\end{array}$ & Tx array & $\begin{array}{c}\text { SLL } \\
(\mathrm{dB})\end{array}$ \\
\hline Wang et al. [3] & Uniform & $\mathrm{Tx}+\mathrm{Rx} /$ Analog beamforming / Retro-reflective & 2.08 & $1 \times 4$ & -15.0 \\
He et al. [4] & Uniform & $\mathrm{Tx}+\mathrm{Rx} /$ Analog beamforming / Retro-reflective & 2.125 & $1 \times 8$ & -2.4 \\
Bae et al. [5] & Uniform & $\mathrm{Tx}+\mathrm{Rx} /$ Analog beamforming / LUT & 5.2 & $4 \times 8$ & -15.0 \\
Takabayashi et al. [6] & Gaussian & Tx only & 5.8 & $1 \times 14$ & -17.7 \\
Mistialustina and Munir [7] & Kaiser & Tx only & 9.5 & $1 \times 7$ & -12.8 \\
Hill and Kelly [8] & Taylor & Tx only & 28 & $1 \times 8$ & -15.2 \\
Mistialustina et al. [9] & Chebyshev & Tx only & 9.3 & $1 \times 5$ & -17.4 \\
This study & Chebyshev & Tx + Rx / Digital beamforming / Far-field approximation & 5.8 & $4 \times 4$ & -19.1 \\
\hline
\end{tabular}




\section{CONCLUSION}

This paper proposes a sidelobe suppression beamforming scheme for an MPT system with PAA. The technique applied to the proposed system involves controlling the magnitude of the signal transmitted from the array antenna. To apply this to a two-dimensional PAA, we described the procedure for obtaining the amplitude distribution matrix $(M \times N)$. Furthermore, to minimize decreased directivity due to the non-uniform amplitude distribution and to lower the SLL, we used the Chebyshev taper for the amplitude distribution.

To verify the proposed scheme, we implemented a digital beamforming system with a $4 \times 4 \mathrm{Tx}$ array and an $\mathrm{Rx}$ at 5.8 $\mathrm{GHz}$. The radiation pattern results while adjusting the amplitude distribution confirmed that the SLL decreased by $7.5 \mathrm{~dB}$ to $-19.1 \mathrm{~dB}$ on the $z x$ plane, and by $4.4 \mathrm{~dB}$ to $-15.9 \mathrm{~dB}$ on the $z y$ plane, compared with uniform distribution. Furthermore, we verified that the received RF power was $20.4 \mathrm{dBm}$, even with sidelobe suppression. This amounted to a reduction of only $0.6 \mathrm{~dB}$ compared with the uniform distribution case. Through a color map, we demonstrated that the area occupied by the sidelobe decreased in the three-directional beamforming experiment. Moreover, compared with other studies, the proposed system has independent and flexible amplitude and phase control. Consequently, the adaptive beamforming system presented in this paper should be capable of delivering efficient and safe MPT by accurately forming a designed beam pattern.

This work was partly supported by the Basic Science Re-search Program of the National Research Foundation of Korea (NRF) funded by the Ministry of Education (No. NRF-2020R1F1A1061826) and partly by an NRF Grant awarded by the Korean government (MSIP) (No. NRF2017R1A5A1015596).

\section{REFERENCES}

[1] K. W. Choi, A. A. Aziz, D. Setiawan, N. M. Tran, L. Ginting, and D. I. Kim, "Distributed wireless power transfer system for Internet of Things devices," IEEE Internet of Things Journal, vol. 5, no. 4, pp. 2657-2671, 2018.

[2] B. J. DeLong, A. Kiourti, and J. L. Volakis, "A radiating near-field patch rectenna for wireless power transfer to medical implants at $2.4 \mathrm{GHz}$," IEEE Journal of Electromagnetics, RF and Microwaves in Medicine and Biology, vol. 2, no. 1, pp. 64-69, 2018.

[3] X. Wang, S. Sha, J. He, L. Guo, and M. Lu, "Wireless power delivery to low-power mobile devices based on retroreflective beamforming," IEEE Antennas and Wireless Propagation Letters, vol. 13, pp. 919-922, 2014.

[4] J. He, X. Wang, L. Guo, S. Shen, and M. Lu, "A distributed retro-reflective beamformer for wireless power transmission," Microwave and Optical Technology Letters, vol. 57, no. 8, pp. 1873-1876, 2015.

[5] J. Bae, S. H. Yi, H. Koo, S. Oh, H. Oh, W. Choi, et al., "LUT-based focal beamforming system using 2-D adaptive sequential searching algorithm for microwave power transfer," IEEE Access, vol. 8, pp. 196024-196033, 2020.

[6] N. Takabayashi, N. Shinohara, and T. Fujiwara, "Array pattern synthesis of flat-topped beam for microwave power transfer system at volcanoes," in Proceedings of 2018 IEEE Wireless Power Transfer Conference (WPTC), Montreal, Canada, 2018, pp. 1-4.

[7] H. Mistialustina and A. Munir, "Characterization of power weighted linear antenna array using Kaiser function in comparison with uniform distribution," in Proceedings of 2020 27th International Conference on Telecommunications (ICT), Bali, Indonesia, 2020, pp. 1-5.

[8] T. A. Hill and J. R. Kelly, "28 GHz Taylor feed network for sidelobe level reduction in $5 \mathrm{G}$ phased array antennas," $M i-$ crowave and Optical Technology Letters, vol. 61, no. 1, pp. 3743, 2019.

[9] H. Mistialustina, M. R. Effendi, and A. Munir, "Radiation characteristics of Chebyshev function-based power weighted linear array antennas influenced by elements configuration," in Proceedings of 2019 IEEE Conference on Antenna Measurements \& Applications (CAMA), Bali, Indonesia, 2019, pp. 1-4.

[10] C. A. Balanis, Antenna Theory: Analysis and Design. Hoboken, NJ: John Wiley \& Sons, 2015.

[11] C. L. Dolph, "A current distribution for broadside arrays which optimizes the relationship between beam width and side-lobe level," Proceedings of the IRE, vol. 34, no. 6, pp. 335-348, 1946.

[12] R. L. Haupt, Antenna Arrays: A Computational Approach. Hoboken, NJ: John Wiley \& Sons, 2010. 


\section{Inho Park}

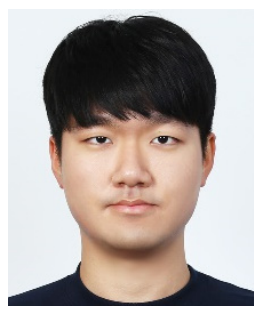

received his B.Sc. degree in electronic engineering from Konkuk University, Seoul, South Korea, in 2021. He is currently pursuing his M.Sc. degree in electronic engineering at the same institute. His current research interests include wireless power transfer and digital RF systems.

\section{Chulhun Seo}

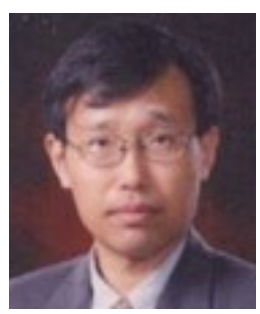

received his B.Sc., M.Sc., and Ph.D. degrees from Seoul National University, Seoul, South Korea, in 1983, 1985, and 1993, respectively. From 1993 to 1995, he was employed by the Massachusetts Institute of Technology (MIT), Cambridge, MA, USA, as a technical staff member, and as a visiting professor from 1999 to 2001. From 1993 to 1997, he was employed by Soongsil University, Seoul, as an assistant professor, where he was also an associate professor from 1997 to 2004. Since 2004, he has been a professor of electronic engineering at Soongsil University. He was the IEEE MTT Korea chapter chairperson from 2011 to 2014. He is the president of the Korean Institute of Electromagnetic Engineering and Science (KIEES) and the dean of the Information and Telecommunications College at Soongsil University. He is the director of the Wireless Power Transfer Research Center, supported by the Korean Government's Ministry of Trade, Industry, and Energy; the Metamaterials Research Center, supported by Basic Research Laboratories (BRL) through an NRF Grant funded by the Ministry of Science, ICT, and Future Planning (MSIP); and the Center for Intelligent Biomedical Wireless Power Transfer, supported by the National Research Foundation of Korea (NRF; grant funded by MSIP). His research interests include wireless technologies, RF power amplifiers, and wireless power transfer using metamaterials.

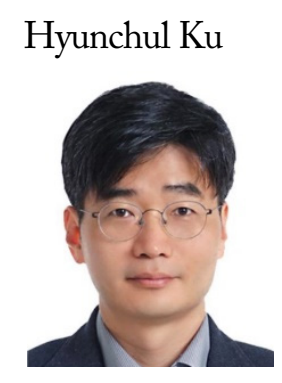

received his B.Sc. and M.Sc. degrees in electrical engineering from Seoul National University, Seoul, South Korea, in 1995 and 1997, respectively, and a $\mathrm{Ph} . \mathrm{D}$. in electrical and computer engineering from the Georgia Institute of Technology, Atlanta, GA, USA, in 2003. From 1997 to 1999, he worked at the Wireless Communication Research Center in KT, Seoul. From 2004 to 2005, he was employed at the Research and Development Laboratory, Mobile Communication Division, Samsung Electronics, Suwon, South Korea. Since 2005, he has worked as a professor at Konkuk University. His research interests include digital RF systems, RF power amplifiers, RF front-end design, and wireless power transfer systems. 